\title{
El lado positivo del emprendedor universitario. Autoestima, satisfacción con la vida y optimismo en estudiantes de México y Bolivia
}

Marco A. Nuñez-Ramírez ${ }^{(1) *}$, Roger A. Banegas-Rivero(2), Berta E. Madrigal-Torres ${ }^{(3)}$ y Cecilia L. Velarde-Flores ${ }^{(4)}$ (1) Instituto Tecnológico de Sonora, Departamento de Ciencias Administrativas, 5 de Febrero 818 Sur, Ciudad Obregón, Sonora, México. (correo-e: marco.nunez@itson.edu.mx).

(2) Universidad Autónoma Gabriel René Moreno, Facultad de Economía, Av. Busch 244, Santa Cruz de la Sierra, Bolivia. (correo-e: aleconomista@gmail.com).

(3) Universidad de Guadalajara, Centro Universitario Sur, Av. Enrique Arreola Silva No. 883, Colonia Centro, 49000, Ciudad Guzmán, México. (correo-e: madrigal@cusur.udg.mx).

(4) El Colegio de Sonora, Obregón 54, 83000, Hermosillo, Sonora, México.

(correo-e: cvelarde@colsonmx.onmicrosoft.com).

* Autor a quien debe ser dirigida la correspondencia.

Recibido Nov. 19, 2019; Aceptado Ene. 14, 2020; Versión final Mar. 22, 2020, Publicado Ago. 2020

\begin{abstract}
Resumen
El objetivo de la presente investigación es comparar la autoestima, optimismo y satisfacción con la vida entre universitarios emprendedores y no emprendedores. Bajo este contexto, la psicología positiva podría ser una perspectiva emergente que permita la comprensión de los atributos que caracterizan al emprendedor universitario. Se empleó una metodología cuantitativa, descriptiva-comparativa y correlacional. Asimismo, se estudió a una muestra conformada por 539 estudiantes universitarios de México y Bolivia. Los resultados obtenidos, a través del empleo de ANOVA de un factor y regresión lineal, reflejaron que los emprendedores dentro del contexto universitario poseen valores más altos de autoestima y optimismo en comparación con estudiantes que no han emprendido. Se concluye que la psicología positiva puede ser una propuesta teórico-metodológica que puede favorecer la formación de futuros emprendedores de la región.
\end{abstract}

\section{The positive side of the college entrepreneur. Self-esteem, satisfaction with life, and optimism in students from Mexico and Bolivia}

\begin{abstract}
The aim of this study is to compare self-esteem, optimism, and satisfaction with life between college entrepreneurs and non-entrepreneurs. In this context, positive psychology could be an emerging perspective that would allow the understanding of attributes that characterize the college entrepreneur. A quantitative, descriptive-comparative and correlational methodology is used. A total of 539 college students from Mexico and Bolivia were considered in the study. The results obtained using one-way ANOVA and linear regression analyses show that entrepreneurs within the university context have higher values for self-esteem and optimism than students who are not entrepreneurs. In conclusion, positive psychology can be a theoreticalmethodological proposal that can favor the education of future entrepreneurs in the countries studied.
\end{abstract}

Keywords: entrepreneurship; positive psychology; satisfaction with life; self-esteem; optimism 


\section{INTRODUCCIÓN}

Comprender el comportamiento del emprendedor juega un rol relevante para estudiar al emprendimiento (Gruber y MacMillan, 2017). Por tal motivo, la psicología se ha convertido en una herramienta valiosa para dilucidar este fenómeno (Frese y Gielnik, 2014). Bajo este contexto destaca la visión que se centra en la personalidad del emprendedor (Zhao et al., 2010), a través de la cual sobresalen dos posturas básicas y opuestas que abordan los rasgos o atributos psicológicos del emprendedor: la negativa y la optimista. La primera hace énfasis en las posibles debilidades de la personalidad de quien inicia una empresa. En este sentido, autores reportan que el emprendedor puede presentar rasgos de psicopatología (Akhtar et al., 2013); mientras que, por otro lado, Freeman et al. (2019) encontraron que los emprendedores muestran diversos problemas que le afectan de manera negativa a su nivel de bienestar, tales como la depresión, trastorno de déficit de atención y abuso de sustancias.

Por otro lado, la visión optimista describe al emprendedor como un individuo con diferentes atributos donde predominan -entre otras- el optimismo (Adomako et., 2016; Trevelyan, 2008), la autoestima (Arora et al., 2013; Laguna, 2013; Mruk, 2008), y la satisfacción con la vida (Hessels et al., 2018). Aquí, se resalta que teóricamente dichos atributos pueden ser explicados por la psicología positiva, la cual en los últimos años ha venido cobrando relevancia dentro del estudio del comportamiento. Esto, debido a que, a diferencia de la psicología tradicional, tal postura se centra en las fortalezas del individuo, poniendo énfasis en los rasgos individuales que pueden influir en el nivel de la calidad de vida a través de las variables mencionadas.

Es importante resaltar que dentro de la presente investigación se considera al emprendedor como un sujeto con la capacidad para generar una nueva empresa (Shane, 2012). Además, a pesar de que no existe un consenso sobre la definición de las variables de estudio por parte de la comunidad académica, para fines de este documento, se concibe a la autoestima como un conjunto de elementos que hacen que un individuo se sienta valioso, auténtico y eficaz (Stets y Burke, 2014); mientras que, en cuanto al optimismo, éste puede ser definido como una expectativa positiva del futuro, lo cual permite que la persona se adapte a su entorno (Trevelyan, 2008). Finalmente, la satisfacción con la vida es entendida como un juicio cognitivo a través del cual un sujeto compara sus propias circunstancias respecto a cierto patrón subjetivo que se considera deseable (Prasoon y Chaturvedi, 2016). A continuación, se expone evidencia empírica que ha puesto hincapié en la relación de dichas variables con la actividad emprendedora.

Primeramente, han surgido estudios cuyos hallazgos proponen que ciertos aspectos relacionados con el comportamiento del emprendedor se encuentran íntimamente asociados con la satisfacción con la vida. Por ejemplo, Arora et al. (2013) reportan que la autoeficacia emprendedora se relaciona de forma relevante con la satisfacción con la vida al estudiar a dichas variables en emprendedores de Estados Unidos. Mientras tanto, Hessels et al. (2018) encontraron que los emprendedores cuentan con niveles más altos de satisfacción con la vida en comparación con empleados; incluso, dichos autores proponen que el autoempleo puede ayudar a elevar los niveles bajos de satisfacción con la vida. Lo anterior, al tratar de relacionar emprendimiento y satisfacción con la vida, refleja dos cosas. Por un lado, existe una relación significativa entre ambos aspectos; mientras que, por el otro, todavía no está clara cómo es dicha asociación: positiva o negativa.

Asimismo, al considerar el rol de la autoestima dentro del comportamiento emprendedor es posible encontrar posiciones opuestas. En primer lugar, desde un punto de vista positivo, se encuentra evidencia empírica que sostiene que la autoestima se asocia de manera significativa con aspectos relacionados con el comportamiento del emprendedor (Arora et al., 2013); incluso la autoestima puede ser un elemento predictor de la intención emprendedora en personas desempleadas (Laguna, 2013). Mientras que, desde una segunda perspectiva, Kets de Vries (1996) sostiene que la necesidad de logro y reconocimiento social pueden ser considerados como elementos descriptivos del emprendedor; quien, a su vez, puede presentar ciertos problemas para regular su nivel autoestima. Es así que, bajo esta perspectiva, muchos emprendedores pueden ser narcisistas y reactivos ante circunstancias adversas. Otro aspecto relevante para comprender al emprendedor es el optimismo, que puede ser entendido como una expectativa positiva del futuro (Trevelyan, 2008). Su importancia radica en que dicha variable favorece a la persistencia del emprendedor, la cual puede ser uno de sus principales distintivos (Adomako et al., 2016). De hecho, Kourstaal et al. (2015) encontraron que los emprendedores son más optimistas que los directivos y empleados. Esto podría servir para explicar por qué quienes inician negocios sobresalen por poseer altos niveles de resiliencia y constancia.

Si bien es cierto el estudio de la psicología del emprendedor ha ido en aumento (Frese y Gielnik, 2014), todavía no se ha logrado caracterizar de manera completa su personalidad, atributos y comportamiento. Por lo tanto, el presente artículo propone que una forma apropiada de conocerlo es a través de la comparación de las características distintivas del emprendedor con otros grupos como los siguientes: 1) personas que no han emprendido, pero desean hacerlo algún día; y 2) quienes no han emprendido ni tienen la intención de 
hacerlo. Para ello se propone realizar el estudio en ambiente específico, es decir, el contexto universitario en Latinoamérica, el cual se describe más adelante.

\section{OTROS ANTECEDENTES}

Según reportes del Monitor Global de Emprendimiento ([GEM], 2018), Latinoamérica posee los niveles más altos en cuanto al nivel de actividad emprendedora en todo el mundo; fenómeno que se observa en economías con menor desarrollo. Bajo este mismo aspecto, Bolivia se ha destacado por obtener el sexto puesto entre los países evaluados, y tercero de la región, considerando la medición del TEA (Tasa de Actividad Emprendedora Temprana), que consiste en el porcentaje de la población entre 18 y 64 años que inicia un negocio; lo anterior, según el Reporte GEM (2014), el cual-como último reporte de este país- es presentado por Querezaju et al. (2015). Mientras que, en el caso de México, durante el 2017, éste se ubicó en el lugar 17 del ranking mundial (GEM, 2018). Cabe señalar que, de acuerdo a dichos rankings, los resultados de TEA para estos países fueron de $27.4 \%$ y $12.98 \%$, respectivamente.

Aunque los números parecen alentadores en cuanto a este ranking, es importante resaltar que otro indicador medido por el GEM (2018) es la autopercepción sobre el emprendimiento, donde tales países no obtuvieron valores tan favorables. Cabe señalar que en dicho estudio se consideran ciertos atributos psicológicos- que determinan a la actividad emprendedora, tales como la percepción de capacidades, percepción de oportunidades, intención emprendedora y miedo al fracaso. Es así que, tanto la parte teórica como los rankings, convergen en que la parte psicológica puede ser un aspecto explicativo que permita desarrollar y direccionar la actividad emprendedora, no solo orientada hacia la generación de negocios como ya se hace en países latinos- sino también hacia la innovación y generación de conocimiento; aspectos que requieren mayor atención en Latinoamérica. Aquí, la participación de la universidad puede jugar un rol importante debido a que en las aulas de clase es posible desarrollar el emprendimiento (Etzkowitz, 2013); sin embargo, es significativo señalar que los estudios que han tratado de explicar, desde un enfoque psicológico, los atributos del emprendedor dentro del contexto universitario son pocos. Esto, a pesar de que dentro de la universidad es posible generar programas y metodologías para desarrollar los atributos del emprendedor en la región (Hernández et al., 2016); modelo que pueden ayudar a desarrollar la intención emprendedora, la cual es entendida como la aspiración a generar una nueva empresa (Zhao et al., 2010).

Es así que un escenario propicio para abordar esta problemática es el contexto universitario. Partiendo de la premisa de que el comportamiento emprendedor no se debe al nacimiento, sino que es también aprendido (López-Núñez et al., 2020; Viinikaine et al, 2017), ciertas investigaciones proponen que la universidad juega un papel relevante para desarrollar las habilidades del emprendedor (Henry et al., 2005). De hecho, en países desarrollados (Estados Unidos, Japón y Noruega) cada vez cobra mayor auge el concepto de universidad emprendedora -entendida como un modelo de universidad que fomenta la creatividad, innovación y autoempleo dentro de sus estudiantes-, la cual, al mismo tiempo que juega un rol significativo como vínculo hacia la economía del conocimiento, también se ha constituido como una parte esencial dentro de la triple hélice -universidad, industria y gobierno-, favoreciendo la innovación y la generación de empleos, al mismo tiempo que añade valor a la sociedad (Etzkowitz, 2013).

No obstante, a pesar que los estudios teóricos y empíricos sobre la universidad emprendedora a nivel mundial han aumentado, los cuales han puesto énfasis en la parte formal [estructura y educación] e informal [actitudes] (Guerrero et al., 2006), en Latinoamérica todavía no se tiene claro cómo intervienen estos elementos en la explicación de los atributos de los emprendedores dentro de la universidad. Es así que el caracterizar al emprendedor universitario juega un papel muy importante para el establecimiento de este modelo educativo en Latinoamérica, debido a que las instituciones de educación superior, dentro de sus planes de estudio, pueden favorecer el desarrollo de atributos que explican al emprendedor, tales como la autoestima, satisfacción con la vida y optimismo. Bajo este contexto, el empleo de la psicología podría arrojar evidencia relevante para poder comprender dichos elementos (Frese y Gielnik, 2014), en especial, al emprendedor universitario, el cual, debido a su contexto, amerita ser estudiado de manera particular. Cabe señalar que en esta investigación se entiende como emprendedor universitario como aquel estudiante de educación superior que, además de cursar sus estudios, ha iniciado su propio negocio.

Como ya se comentó anteriormente, desde un enfoque teórico, esta investigación ha puesto énfasis en el estudio de ciertos atributos del emprendedor universitario, tales como la autoestima, satisfacción con la vida y optimismo, desde la psicología positiva. Esto, con el fin de arrojar evidencia empírica que pueda servir de insumo para el establecimiento del modelo de universidad emprendedora en Latinoamérica, región que destaca a nivel mundial por tener los niveles más altos de actividad emprendedora (GEM, 2018). A través de una muestra obtenida de estudiantes de México y Bolivia se busca caracterizar dichos atributos dentro de los emprendedores universitarios y, posteriormente, diferenciarlos respecto a otros estudiantes que no se sienten atraídos por la generación de empresas. A partir de tales supuestos surge las siguientes preguntas 
de investigación: ¿existen diferencias significativas entre en el nivel de autoestima, optimismo y satisfacción con la vida entre universitarios emprendedores y no emprendedores? Si es así, ¿el emprendedor universitario presenta niveles más altos de autoestima, optimismo y satisfacción con la vida respecto a los estudiantes que no han emprendido? Para atender a dicha cuestiones se plantea la siguiente hipótesis: $H 1$. Los "emprendedores universitarios" presentan valores más altos de autoestima, satisfacción con la vida y optimismo respecto a "estudiantes no emprendedores que no desean emprender" y "estudiantes no emprendedores, pero que desean emprender a futuro".

Aquí, se parte de la premisa que sostiene que la psicología positiva -a diferencia de la psicología tradicional- se fundamenta en las fortalezas que permiten el desarrollo del ser humano (Seligman y Pawelski, 2003). Por lo tanto, el emprendimiento puede ser explicado por medio de variables que componen a esta postura teórica (Juhdi y Juhdi, 2013); a través de esto, es posible destacar a la satisfacción con la vida, el optimismo y la autoestima. No obstante, aunque existen estudios que han puesto énfasis en las diferencias del nivel de dichas variables al comparar muestras de emprendedores y empleados, todavía no está claro cómo estos atributos pueden impactar dentro de la intención para emprender.

Por otro lado, la presente investigación se fundamenta en dos premisas básicas: 1) los rasgos de la personalidad y ciertos aspectos propios del sujeto pueden afectar a la intención emprendedora (Zhao et al., 2010; López-Núñez et al., 2020); 2); existe una controversia teórica al explicar los atributos del emprendedor, pues destacan quienes ponen énfasis en sus atributos positivos (Adomako et al., 2016; Arora et al., 2013; Laguna, 2013; Trevelyan, 2008; Hessels et al., 2018), y otros que se centran en los negativos (Akhtar et al., 2013; Freeman et al., 2019). De tal manera, atendiendo a la psicología positiva, la cual se centra en las fortalezas de los individuos (Seligman y Pawelski, 2003), ¿es posible que variables de la psicología positiva como la autoestima, satisfacción con la vida y el optimismo puedan explicar a la intención emprendedora? Esto considerando que tal postura puede favorecer a la comprensión del emprendedor (Juhdi y Juhdi, 2013). Asimismo, ¿es posible que otras variables generales de la persona (socioeconómicas) pueden favorecer a la intención para emprender? Para dar respuesta a dichas premisas, se plantea la segunda hipótesis de estudio: $\mathrm{H}_{2}$. La satisfacción con la vida, el optimismo y satisfacción con la vida, así como variables socioeconómicas afectan a la intención para emprender". Las variables socioeconómicas son descritas en la Tabla 1.

\section{MATERIALES Y MÉTODOS}

En esta sección se muestra la metodología empleada, así como los sujetos de estudio, las características psicométricas de los instrumentos de medición y el procedimiento utilizado. En el primero de los casos, se resalta que, con el fin de cumplir el objetivo planteado, se realizó una investigación de tipo cuantitativa, con un alcance descriptivo-comparativo y correlacional, cuyo diseño fue no experimental.

\section{Instrumentos de medición}

La primera pregunta del cuestionario fue la siguiente: 1) ¿Planeas emprender después de egresar de la universidad? La cual fue medida a través de una escala Likert de 4 puntos, cuyos valores van de 1(Muy improbable) a 4(Muy probable). Dicha interrogante fue adaptada de Lüthje y Franke (2003), que, según estos autores, se orienta a describir la intención emprendedora. Posteriormente, con el fin de medir las variables de estudio, se utilizaron tres instrumentos de medición. A continuación, se muestran sus antecedentes y sus respectivas escalas, así como su validez y confiabilidad.

Autoestima. Se empleó una adaptación al español del Rosenberg Self-esteem Scale o Escala de Autoestima de Rosenberg (Rosenberg, 1965), el cual consta de 10 ítems con en una escala Likert 4 con los siguientes valores: 1 (Muy en desacuerdo), 2 (En desacuerdo), 3 (De acuerdo) y 4 (Muy de acuerdo). El cuestionario se conforma de dos secciones. La primera se encuentra integrada por cinco afirmaciones, mientras que la segunda, por cinco negaciones. Cabe señalar que, para el procesamiento estadístico de los resultados de la segunda sección, los valores fueron invertidos. Respecto a la validez de constructo, por medio de análisis factorial con rotación Varimax, se hallaron valores para la prueba Kaiser-Meyer-Olkin $(\mathrm{KMO}=.827)$ y Esfericidad de Bartlett $\left(\mathrm{X}^{2}=1317 ; g l=45 ; p<0.001\right)$, encontrando que las dos subdimensiones explican el $50.17 \%$ de la varianza. Finalmente, en cuanto a la confiabilidad, se obtuvo un valor alpha de .793 para toda la variable; resultados similares fueron encontrados para las dos subescalas (positiva $[\alpha=.751]$ y negativa $[\alpha=.726]$ ). Para efectos de este estudio, se analizó a la autoestima de manera unidimensional; esto con el fin de que todas las variables sean homogéneas.

Satisfacción con la vida. Para la medición de esta variable se utilizó una adaptación al español del cuestionario conocido como The Satisfaction with Life Scale (SWLS) o Escala de satisfacción con la vida, propuesto por Diener et al. (1985), el cual está compuesto por cinco ítems en escala Likert 5: 1 (Totalmente en desacuerdo), 2 (En desacuerdo), 3 (Ni de acuerdo ni en desacuerdo), 4 (De acuerdo) y 5 (Totalmente de 
acuerdo). Esta variable unidimensional mostró valores favorables en la medición de la validez de constructo $\left(\mathrm{KMO}=.810\right.$; Esfericidad de Bartlett $\left[\mathrm{X}^{2}=649 ; g l=10 ; p<0.001\right)$, con una varianza total explicada de $52.79 \%$ con un solo factor. Su fiabilidad fue de .769 a través del estadístico alpha de Cronbach.

Optimismo. Se adaptó la versión española de la subescala de General Mood (Estado de Ánimo General) que pertenece al instrumento Emotional Quotient Inventory o Inventario de Cociente Emocional (EQ-i, YV) encontrada en Oliva et al. (2011 citando a Bar-On y Parker, 2000). Éste se compone por ocho ítems con escala Likert 5 con los siguientes valores: 1 (Nunca), 2 (Rara vez), 3 (Regularmente), 4 (Con frecuencia) y 5 (Siempre). Asimismo, su validez fue representada a través de los siguientes valores: $\mathrm{KMO}=0.896$; Esfericidad de Bartlett $\left(X^{2}=2291 ; g l=28 ; p<0.001\right)$, donde un solo factor explica el $59 \%$ de la varianza. En cuando a la confiabilidad, los autores reportan un valor $(\alpha=0.91)$; resultado similar al encontrado en la presente investigación $(\alpha=0.894)$.

Tabla 1: Caracterización de la muestra $(n=539)$

\begin{tabular}{|c|c|c|}
\hline Características & $n$ & $\%$ \\
\hline \multicolumn{3}{|l|}{ País } \\
\hline México & 263 & 48.8 \\
\hline Bolivia & 276 & 51.2 \\
\hline \multicolumn{3}{|l|}{ Género } \\
\hline Femenino & 318 & 59.0 \\
\hline Masculino & 221 & 41.0 \\
\hline Edad & $M=21.19$ & $D E=2.88$ \\
\hline \multicolumn{3}{|l|}{ Licenciatura } \\
\hline Administración & 129 & 23.9 \\
\hline Contaduría & 66 & 12.2 \\
\hline Economía y Finanzas & 52 & 9.6 \\
\hline Turismo & 46 & 8.5 \\
\hline Otro & 55 & 10.3 \\
\hline Ingeniería Comercial & 55 & 10.2 \\
\hline Ingeniería Financiera & 81 & 15.0 \\
\hline Agronegocios & 12 & 2.2 \\
\hline Ingeniería Ambiental & 6 & 1.1 \\
\hline Economía & 30 & 5.6 \\
\hline Ingeniería Industrial & 7 & 1.3 \\
\hline \multicolumn{3}{|l|}{ Semestre } \\
\hline Primero & 35 & 6.5 \\
\hline Segundo & 88 & 16.3 \\
\hline Tercero & 44 & 8.2 \\
\hline Cuarto & 54 & 10.0 \\
\hline Quinto & 61 & 11.3 \\
\hline Sexto & 25 & 4.6 \\
\hline Séptimo & 54 & 10.0 \\
\hline Octavo & 72 & 13.4 \\
\hline Noveno & 7 & 1.3 \\
\hline Otro & 99 & 18.4 \\
\hline \multicolumn{3}{|l|}{ Status emprendedor } \\
\hline Emprendedor & 154 & 28.6 \\
\hline No emprendedor sin deseo de emprender a futuro & 194 & 36.0 \\
\hline No Emprendedor con intención de emprender a futuro & 191 & 35.4 \\
\hline
\end{tabular}

\section{Participantes}

Se obtuvo una muestra no probabilística por conveniencia conformada por 539 estudiantes de universidades de México y Bolivia, de los cuales 154 eran emprendedores (28.6\%), 194 se describieron como no "emprendedores, pero con intenciones de emprender en un futuro (36\%), y 191 estudiantes dijeron que se consideran "No emprendedores con intención de emprender a futuro". La caracterización puede ser observada en la Tabla 1. La razón de haber tomado como prueba empírica a estudiantes de dichos países se fundamenta en lo siguiente: 1) estos países forman parte de la misma región donde se comparten 
similitudes culturales (lengua, religión, valores); 2) ambos destacan por sus altos niveles de TEA a nivel mundial según el GEM; 3) en esta Latinoamérica no existen muchos estudios similares que involucren a la psicología positiva para comprender los atributos del emprendedor en varios países; y finalmente, 4) se pretende saber cómo la nacionalidad afecta en la intención emprendedora.

\section{Procedimiento}

Dichos instrumentos de medición cuantitativos fueron autoadministrados a estudiantes que accedieron a participar en el estudio durante 2018 y, además, se contó con la aprobación de las autoridades universitarias correspondientes. Se contó con el apoyo de una universidad pública del noroeste de México, mientras que, en el caso de Bolivia, se realizó la investigación en dos universidades privadas y una pública, que están ubicadas dentro del Departamento de Santa Cruz, destacando que todas las anteriores se encentraban en sector urbano. Posteriormente, con el propósito de verificar las hipótesis de estudio propuestas, fueron empleadas las pruebas ANOVA de un factor con Post Hoc Bonferroni y regresión lineal.

\section{RESULTADOS}

Las presentaciones de los resultados y la discusión son presentadas partiendo de cada una de las hipótesis planteadas. Primeramente, la muestra se subdividió en tres grupos, cuya descripción es la siguiente: 1) estudiantes universitarios emprendedores ([Emprendedor] $n=154$ ); 2) estudiantes universitarios no emprendedores y que no desean emprender a futuro ([No emprendedor] $n=194$ ); y 3 ) estudiantes universitarios no emprendedores, pero que desean emprender luego de egresar ([Futuro emprendedor] $n=$ 191).

Dentro de $H_{1}$ se planteó que existen diferencias significativas respecto a la medición de autoestima, satisfacción con la vida y optimismo entre estos tres grupos. Es así que, considerando a dichos aspectos como atributos positivos del emprendedor, se postuló que los resultados de los emprendedores universitarios serían mayores respecto a quienes no lo son. Para ello, se empleó la prueba ANOVA de un factor donde se encontró que efectivamente existen diferencias significativas entre las muestras de estudio, siendo que los valores más altos fueron para quienes han iniciado un negocio en cuanto a la medición de la autoestima ([Emprendedor: $M=3.35 ; D E=0.411$ ]; [No Emprendedor: $M=3.21 ; D E=0.450$ ]; [Futuro emprendedor: $M=3.33 ; D E=0.451] ; F=5.216 ; p<0.01$ ), y optimismo ([Emprendedor: $M=4.28 ; D E=$ 0.620]; [No Emprendedor: $M=3.93 ; D E=0.760$ ]; [Futuro emprendedor: $M=4.17 ; D E=0.618$ ]; $F=12.707$; $p<0.001$ ). Asimismo, dichos hallazgos fueron confirmados por medio de la prueba Post Hoc Bonferroni, donde es posible observar que los resultados del emprendedor fueron mayores solamente en cuanto a las variables de autoestima y optimismo (Ver Tabla 3).

Sin embargo, es importante señalar que no existieron diferencias significativas entre dichos grupos en cuanto a la satisfacción con la vida. Además, se realizó la prueba T de Student para comparar las variables de acuerdo a dos grupos: emprendedor $(n=154)$ y futuro emprendedor $(n=191)$, donde no fue posible encontrar diferencias significativas entre estos en ninguno de los casos. Es así que, considerando ambos métodos de comparación de medias, es posible postular que existen discrepancias reales entre quien ya ha emprendido y quien no tiene la intención de hacerlo. Tal hallazgo permite ver que la intención emprendedora juega un rol importante para describir los atributos del emprendedor.

Tabla 2: ANOVA de un factor según Status emprendedor

\begin{tabular}{|c|c|c|c|c|c|c|c|}
\hline & & $\begin{array}{l}\text { Suma de } \\
\text { Cuadrados }\end{array}$ & $g l$ & $\begin{array}{c}\text { Media } \\
\text { Cuadrática }\end{array}$ & $F$ & $p$ & $\eta^{2}$ \\
\hline \multirow{3}{*}{ Autoestima } & Entre grupos & 2.013 & 2 & 1.006 & 5.216 & 0.006 & 0.020 \\
\hline & Dentro de grupos & 103.226 & 535 & 0.193 & & & \\
\hline & Total & 105.239 & 537 & & & & \\
\hline \multirow{3}{*}{ Satisfacción con la vida } & Entre grupos & 2.512 & 2 & 1.256 & 2.579 & 0.077 & 0.009 \\
\hline & Dentro de grupos & 260.561 & 535 & 0.487 & & & \\
\hline & Total & 263.073 & 537 & & & & \\
\hline \multirow{3}{*}{ Optimismo } & Entre grupos & 11.522 & 2 & 5.761 & 12.707 & 0.000 & 0.045 \\
\hline & Dentro de grupos & 242.560 & 535 & 0.453 & & & \\
\hline & Total & 254.082 & 537 & & & & \\
\hline
\end{tabular}


Tabla 3: Datos descriptivos con Post Hoc Bonferroni

\begin{tabular}{|l|c|c|r|r|r|r|c|}
\hline \multicolumn{1}{|c|}{ Variables } & \multicolumn{2}{c|}{$\begin{array}{c}\text { (1) Emprendedor } \\
(n=154)\end{array}$} & \multicolumn{2}{c|}{$\begin{array}{c}\text { (2) No emprendedor } \\
(n=194)\end{array}$} & \multicolumn{2}{c|}{$\begin{array}{c}\text { (3) Futuro emprendedor } \\
(n=191)\end{array}$} & \\
\hline & $M$ & $D E$ & $M$ & $D E$ & $M$ & \multicolumn{1}{c|}{$D$} & Post Hoc \\
\hline Autoestima & 3.35 & 0.411 & 3.21 & 0.450 & 3.33 & .451 & $1>2,2<3$ \\
\hline Satisfacción con la vida & 3.75 & 0.716 & 3.58 & 0.672 & 3.66 & .709 & $1=2=3$ \\
\hline Optimismo & 4.28 & 0.620 & 3.93 & 0.760 & 4.17 & .618 & $1>2,2<3$ \\
\hline
\end{tabular}

Por otro lado, respecto a $\mathrm{H}_{2}$, se evaluó la asociación entre la intención emprendedora con las variables de estudio. En un primer momento, en la Tabla 4 se puede observar que, mediante la correlación de Pearson se encontraron resultados significativos en todos los casos $(p<0.01)$. Posteriormente, se llevó a cabo regresión lineal donde se formularon cuatro modelos de acuerdo a las variables explicativas: 1) variables control; 2) satisfacción con la vida; 3) optimismo; y 4) autoestima. Cabe señalar que las variables control empleadas fueron género, país de origen, licenciatura y edad, donde se encontró que solo el país y la licenciatura cursada tuvieron algún efecto sobre la intención emprendedora. Además, la variable dependiente solo fue afectada significativamente por la satisfacción con la vida ( $\beta=156 ; p<0.001$ ) y el optimismo ( $\beta=162 p<0.01$ ); lo cual no sucedió en el caso de la autoestima. Asimismo, se resalta que dentro de la regresión no se encontraron problemas de colinealidad. Esto es corroborado en la correlación y el factor de inflación de la varianza (VIF). Además, es de llamar la atención que los resultados de la $\mathrm{R}^{2}$, aunque fueron significativos, mostraron niveles bajos de explicación en todos los modelos.

Tabla 4: Correlación de las variables $(n=539)$

\begin{tabular}{|l|r|r|r|r|}
\hline & 1 & \multicolumn{1}{|c|}{2} & \multicolumn{1}{|c|}{3} & \multicolumn{1}{c|}{4} \\
\hline 1. Satisfacción con la vida & 1 & & & \\
\hline 2. Optimismo & $.596^{* *}$ & 1 & & \\
\hline 3. Autoestima & $.541^{* *}$ & $.590^{* *}$ & 1 & \\
\hline 4. Intención emprendedora & $.151^{* *}$ & $.191^{* *}$ & $.153^{* *}$ & 1 \\
\hline
\end{tabular}

Tabla 5. Regresión lineal teniendo a la intención emprendedora como variable dependiente

\begin{tabular}{|c|c|c|c|c|}
\hline Variables & Modelo 1 & Modelo 2 & Modelo 3 & Modelo 4 \\
\hline Variables control & & & & \\
\hline Género & $\begin{array}{r}.038 \\
.087)\end{array}$ & $\begin{array}{r}.028 \\
(.087)\end{array}$ & $\begin{array}{r}.022 \\
(.086)\end{array}$ & $\begin{array}{r}.024 \\
(.086)\end{array}$ \\
\hline País & $-.144^{*}$ & -.101 & $-.092^{*}$ & $-.077^{*}$ \\
\hline & (.116) & $(.117)$ & $(.116)$ & $(.118)$ \\
\hline Licenciatura & $.165^{* \star}$ & $.174^{\star *}$ & $.171^{\star *}$ & $.176^{\star \star}$ \\
\hline & (.018) & $(.018)$ & $(.018)$ & (.018) \\
\hline Edad & $\begin{array}{r}-.014 \\
(.80)\end{array}$ & $\begin{array}{r}-.009 \\
(.80)\end{array}$ & $\begin{array}{r}-.027 \\
(.80)\end{array}$ & $\begin{array}{c}-.032 \\
(.80)\end{array}$ \\
\hline Satisfacción con la vida & & $\begin{array}{r}.156^{\star \star \star} \\
(.064)\end{array}$ & $\begin{array}{r}.060 \\
(.078)\end{array}$ & $\begin{array}{r}.042 \\
(.080)\end{array}$ \\
\hline Optimismo & & & $\begin{array}{l}.162^{* *} \\
(.077)\end{array}$ & $\begin{array}{l}.131^{*} \\
(.083)\end{array}$ \\
\hline Autoestima & & & & $\begin{array}{r}.079 \\
(.128)\end{array}$ \\
\hline $\mathrm{R}^{2}$ & .018 & .040 & .056 & .060 \\
\hline $\mathrm{R}^{2}$ ajustado & .011 & .031 & .046 & .047 \\
\hline$F$ & $2.455^{\star}$ & $11.939^{* \star \star}$ & $9.302^{*}$ & 1.881 \\
\hline VIF & 1.031 & 1.124 & 1.590 & 1.842 \\
\hline
\end{tabular}

\section{DISCUSIÓN}

El emprendedor universitario debe ser entendido dentro de su contexto. Éste, además de ser un estudiante de nivel superior, también es individuo que ha decido generar su propio negocio. Aunque no se tenga total certeza sobre cómo pudo haber influido la educación en estas personas para tomar la decisión de emprender, cada vez se tiene más claro que la universidad emprendedora juega un rol importante para el desarrollo del emprendimiento a nivel mundial (Etzkowitz, 2013). Partiendo de la premisa de que el 
emprendedor puede formarse dentro de la universidad, surgen muchas incógnitas relacionadas hacia la comprensión del cómo el sistema educativo puede desarrollar todos los atributos que requiere un emprendedor para ser exitoso. Esto muestra que todavía falta mucho por estudiar dentro de este campo. Además, tal visión se vuelve más imperante cuando estudios han propuesto que la universidad ha jugado un papel relevante para incentivar y capacitar a los emprendedores (Ver Guerrero et al., 2006).

En la actualidad, la universidad se está convirtiendo en un factor central para formar y capacitar a los emprendedores en países como México (Hernández et al., 2016) y Bolivia (Querezaju et al., 2015). Por lo tanto, se requiere de más metodologías y estrategias que permitan a los emprendedores universitarios desarrollar la creatividad y la innovación. Esto se vuelve un elemento más destacado al considerar que esta región presenta carencias en cuanto a la generación de nuevos productos dentro de la actividad emprendedora (GEM, 2018).

Al tratar de probar las dos hipótesis de estudio, esta investigación refleja que una metodología emergente que puede favorecer al desarrollo de los atributos del emprendedor, es la psicología positiva. En este sentido, los hallazgos muestran que el emprender se asocia de manera significativa con el optimismo y la autoestima. Esto concuerda con los descubrimientos de Kourstaal et al. (2015), quienes encontraron que los emprendedores son más optimistas que los empleados y directivos. Asimismo, estos resultados soportan empíricamente otros estudios, donde se hallaron que dentro de los atributos del emprendimiento destacan la autoestima (Arora et al., 2013; Laguna, 2013), y el optimismo (Adomako et al., 2016; Trevelyan, 2008).

Asimismo, la intención emprendedora fue explicada a través de la satisfacción con la vida y el optimismo, incluso el país de origen y la carrera de estudio, lo cual confirma dos cosas: 1) la primera variable, aunque no fue significativa al comparar los grupos, sí tiene un rol explicativo en la intención para emprender, tal como lo propone Hessels et al. (2018); 2) aun cuando los países latinoamericanos mantienen similitudes en cuanto a cultura y lengua, y aunque ambos -en diferentes reportes del GEM- han destacado por su TEA, faltan más elementos para diferenciar sobre cuál es el rol que juega la universidad para la formación de emprendedores.

Los hallazgos coinciden con la psicología positiva, la cual sostiene que ciertos aspectos del individuo afectan a la calidad de vida, tales como la satisfacción con la vida, optimismo y autoestima. Asimismo, en las Tablas 4 y 5 , es posible observar lo siguiente: 1) las variables se asociaron de manera positiva y significativa a través del empleo de correlación de Pearson; 2) se encontró que la intención para emprender puede ser explicada a través de la nacionalidad, soportando empíricamente lo reportado por la metodología del GEM (2018), la cual, ha reportado que el emprendimiento depende, al mismo tiempo, tanto de atributos individuales como la intención, así como de aspectos sociales y culturales de cada país; 3) además, se destaca que la licenciatura cursada puede afectar en la intención emprendedora, debido a que, como indica Flores-Aguilar (2019), éste es un factor que influye dentro de la actividad emprendedora; 4) finalmente, tanto la satisfacción con la vida como el optimismo pueden ser consideradas como variables predictoras de la intención emprendedora. Esto último concuerda con la postura que el emprendimiento puede ser explicado a través de fortalezas del ser humano (Adomako et al., 2016; Trevelyan, 2008).

Por otro lado, aunque ciertos autores propusieron que la satisfacción con la vida puede ser uno de los principales atributos que distinguen al emprendedor (Arora et al., 2013; Hessels et al., 2018), dentro del estudio no se encontraron diferencias significativas al comparar emprendedores universitarios y estudiantes que no lo son. Si bien es cierto que algunos elementos de psicología positiva pueden ser empleados para comprender de una mejor manera las fortalezas del emprendedor (Juhdi y Juhdi, 2013), falta más evidencia empírica que permita precisar cuáles variables diferencian y definen a los emprendedores dentro del contexto universitario. Esto debido a que estudios denotan la necesidad de mayor claridad en cuanto a los atributos que caracterizan al emprendedor. Por ejemplo, Trevelyan (2008) encontró que el optimismo se asocia de manera significativa con la actividad emprendedora, lo cual no sucedió entre el exceso de seguridad y actividad emprendedora; variables que, al ser consideradas como fortalezas, podrían ser incluidas dentro de los postulados de la psicología positiva. Por lo tanto, a través del fomento de las fortalezas del ser humano, esta postura podría convertirse en una propuesta teórico-metodológica que pueda intervenir favorablemente en la formación de futuros emprendedores de la región, los cuales no solo sean capaces de fundar empresas, sino que también innoven dentro de la sociedad del conocimiento.

Aunque los hallazgos aquí mostrados no pueden generalizarse debido al tipo de muestreo empleado -no probabilístico-, estos arrojan información relevante para mejorar la comprensión del emprendedor universitario en México y Bolivia. Es así que, sería conveniente asociar a estas variables con otros atributos del emprendedor que son estudiados por la psicología positiva, tales como la autoeficacia, seguridad, resiliencia, bienestar subjetivo, entre otras. Esto, con el propósito de disponer una visión más amplia de la psicología del emprendedor universitario. Además, los resultados reflejan que los atributos psicológicos del emprendedor universitario son elementales para su desarrollo. Ello conlleva retos dentro de su formación en 
todos los contextos. Asimismo, se observa que el establecimiento del modelo de universidad emprendedora está aumentando en la región.

\section{CONCLUSIONES}

El presente documento aborda el vacío teórico y empírico del estudio de los atributos psicológicos del emprendedor universitario, cuyas conclusiones y aportación se pueden representar de la siguiente manera: 1) existen diferencias perceptibles de la autoestima y optimismo al comparar a los emprendedores universitarios con quienes no lo son en estudiantes de Bolivia y México; 2) los emprendedores mostraron niveles más altos de autoestima y optimismo; 3) no se encontraron diferencias significativas en cuanto a la satisfacción con la vida; 4) la intención emprendedora fue influenciada por la satisfacción con la vida, el optimismo y el país de origen; 5) los hallazgos aquí descritos se añaden a un corpus teórico y empírico que trata de comprender a los atributos psicológicos que definen al emprendedor; 6) esta investigación permite la caracterización de ciertos atributos como la autoestima, optimismo y satisfacción con la vida del emprendedor universitario en esto países latinoamericanos estudiados.

\section{REFERENCIAS}

Adomako, S., Danso, A., y otros dos autores, Entrepreneurs' Optimism, Cognitive Style and Persistence, https://doi.org/10.1108/IJEBR-07-2015-0158, International Journal of Entrepreneurial Behavior \& Research, 22(1), 84108 (2016).

Akhtar, R., Ahmetoglu, G. y Chamorro-Premuzic, T., Greed is Good? Assessing the Relationship between Entrepreneurship and Subclinical Psychopathy, https://doi.org/10.1016/j.paid.2012.10.013, Personality and Individual Differences, 54(3), 420-425 (2013).

Arora, P., Haynie, J. M. y Laurence, G.A., Counterfactual Thinking and Entrepreneurial Self-Efficacy: The Moderating Role of Self-Esteem and Dispositional Affect, https://doi.org/10.1111/j.1540-6520.2011.00472.x, Entrepreneurship. Theory and Practice, 37(2), 359-385 (2013).

Diener, E., Emmons, R.A., Larsen, R. y Griffin, S., The Satisfaction with Life Scale. https://doi.org/10.1207/s15327752jpa4901_13, Journal of Personality Assessment, 49(1), $71-75$ (1985).

Etzkowitz, H., Can a Teaching University be an Entrepreneurial University? Civic Entrepreneurship and the Formation of a Cultural Cluster in Ashland, Oregon, Working paper, Birkbeck College, University of London, UK (2013).

Flores-Aguilar, E., Diseño de un Centro para Emprendedores en una Escuela Profesional de Ingeniería Aplicando el Modelo Lean Canvas, http://dx.doi.org/10.4067/S0718-50062019000600151, Formación Universitaria, 12(6), 151-166 (2019).

Freeman, M.A., Staudenmaier, P.J., y otros dos autores, The prevalence and Co-occurrence of Psychiatric Conditions among Entrepreneurs and their Families, https://doi.org/10.1007/s11187-018-0059-8, Small Business Economics, 53(2), 323-342 (2019).

Frese, M., y Gielnik, M.M., The Psychology of Entrepreneurship, https://doi.org/10.1146/annurev-orgpsych-031413091326, Annual Review of Organizational Psychology and Organizational Behavior, 1, 413-438 (2014).

Global Entrepreneurship Monitor (GEM), Global Report 2017/18, Global Entrepreneurship Research Association (GERA) (2018).

Gruber, M., y MacMillan, I.C., Entrepreneurial Behavior: A Reconceptualization and Extension based on Identity Theory, https://doi.org/10.1002/sej.1262, Strategic Entrepreneurship Journal, 11(3), 271-286 (2017).

Guerrero, M., Urbano, D. y Kirby, D., A Literature Review on Entrepreneurial Universities: An Institutional Approach, Working paper, (No.608) Business Economic Department, Autonomous University of Barcelona (2006).

Henry, C., Hill, F., y Leitch, C., Entrepreneurship Education and Training: Can Entrepreneurship be Taught? Part I, https://doi.org/10.1108/00400910510586524, Education + Training, 47(2), 98-111(2005).

Hernández, K.E., González, L. y Herrera, M., Reporte Regional 2015, México, Tecnológico de Monterrey/Global Economic Monitor (2016).

Hessels, J., Arampatzi, E., y otros dos autores, Life Satisfaction and Self-employment in Different Types of Occupations, https://doi.org/10.1080/13504851.2017.1361003, Applied Economics Letters, 25(11), 734-740 (2018).

Juhdi, N.H. y Juhdi, N., Entrepreneurial Success from Positive Psychology View, 4th international conference on business and economic research, 285-295, Indonesia, ISBN: 978-967-5705-10-6, (2013).

Kets de Vries, M.F., The Anatomy of the Entrepreneur: Clinical Observations, https://doi.org/10.1177/001872679604900701, Human Relations, 49(7), 853-883 (1996).

Laguna, M., Self-efficacy, Self-esteem, and Entrepreneurship among the Unemployed, https://doi.org/10.1111/j.15591816.2012.00994.x, Journal of Applied Social Psychology, 43(2), 253-262 (2013). 
López-Núñez, M. I., Rubio-Valdehita, S., Aparicio-García, M.E., Díaz-Ramiro, E.M., Are Entrepreneurs born or made? The influence of personality, https://doi.org/10.1016/j.paid.2019.109699, Personality and Individual Differences, 154, 1-5 (2020).

Lüthje, C., y Franke, N., The 'Making' of an Entrepreneur: Testing a Model of Entrepreneurial Intent among Engineering Students at MIT, https://doi.org/10.1111/1467-9310.00288, R\&D Management, 33(2), 135-147 (2003).

Mruk, C.J., The Psychology of Self-Esteem: A Potential Common Ground for Humanistic Positive Psychology and Positivistic Positive Psychology, https://doi.org/10.1080/08873260802111176, The Humanistic Psychologist, 36(2), 143158 (2008).

Oliva, A., Antolín, L., y otros cuatro autores, Instrumentos para la Evaluación de la Salud Mental y el Desarrollo Positivo Adolescente y los Activos que lo Promueven, Junta de Andalucía. Consejería de Salud. ISBN: 978-84-694-4377-4, España (2011).

Prasoon, R. y Chaturvedi, K.R. Life Satisfaction: A Literature Review, International Journal of Management Humanities and Social Sciences, 1(2), 25-32 (2016).

Querezaju, M., Zavaleta, D. y Mendizabal, J., Global Entrepreneurship Monitor. Reporte Nacional Bolivia 2014, Escuela de la Producción y la Competitividad de la Universidad Católica Boliviana "San Pablo", Bolivia (2015).

Rosenberg, M., Society and the Adolescent Self-image, Princeton University Press, Princeton, NJ (1965).

Seligman, M.E.P. y Pawelski, J., Positive Psychology: FAQs, Psychological Inquiry, 14(2), 159-163 (2003).

Shane, S., Reflections on the 2010 AMR Decade Award: Delivering on the Promise of Entrepreneurship as a Field of Research, https://doi.org/10.5465/amr.2011.0078, Academy of Management Review, 37(1), 10-20 (2012).

Stets, J.A. y Burke, P.J., Self-esteem and Identities, Sociological Perspectives, https://doi.org/10.1177/0731121414536141, 57(4), 409-433 (2014).

Trevelyan, R., Optimism, Overconfidence and Entrepreneurial Activity, https://doi.org/10.1108/00251740810890177, Management Decision, 46(7), 986-1001 (2008).

Viinikainen, J., Heineckb, G. y otros cuatro autores, Born Entrepreneurs? Adolescents' Personality Characteristics and Entrepreneurship in Adulthood, https://doi.org/10.1016/j.jbvi.2017.05.001, Journal of Business Venturing Insights, 8, 9-12 (2017).

Zhao, H., Seibert, S.E. y Lumpkin, G.T., The Relationship of Personality to Entrepreneurial Intentions and Performance: A Meta-Analytic Review, https://doi.org/10.1177/0149206309335187, Journal of Management, 26(2), $381-404$ (2010). 